\title{
Limitations of patient triggered ventilation in neonates
}

\author{
A MitChelL, A GREENOUGH, AND M HIRD \\ Department of Child Health, King's College Hospital, London
}

SUMMARY The practicality of long term patient triggered ventilation using airflow changes was assessed in 22 infants with a median gestational age of 29 weeks (range 25-33 weeks). Inflation time during patient triggered ventilation was limited to 0.4 seconds or less. Initially it was associated with improvements in oxygenation in most infants. Patient triggered ventilation was maintained till final extubation in 13 of the infants without complications. Only one infant developed a pneumothorax, but in the remaining eight infants (who tended to be less mature $(p<0.01))$ it had to be discontinued after only a few hours. Predictors of failure of patient triggered ventilation at one hour were both a lack of improvement in oxygenation, and a relatively slow triggering rate that was related to gestational age. We conclude that long term patient triggered ventilation is practical for preterm neonates, but only for those more mature than 28 weeks' gestational age.

Patient triggered ventilation has been used in preterm infants with success. ${ }^{1-3} \mathrm{~A}$ variety of triggering mechanisms have been used including detection of inspiratory flow changes at the airway, which has been shown to be reliable even in infants of very low birth weight. ${ }^{3}$

Change to patient triggered ventilation from conventional mode has been associated with immediate improvements in oxygenation, particularly during the recovery phase of respiratory distress syndrome. ${ }^{3}$ In those preliminary studies that investigated the feasibility of patient triggered ventilation in the very preterm neonate it was maintained for short periods only. The aim of this study was to assess the practicality of long term patient triggered ventilation in preterm neonates and to examine possible limitations.

\section{Patients and methods}

Twenty two infants, with a median gestational age of 29 weeks (range 25-33) and birth weight of $1450 \mathrm{~g}$ (range 560-1910) were entered into the study. Their median postnatal age was 30 hours (range 3-120). All infants included in the study were being ventilated because of respiratory distress syndrome or respiratory distress associated with severe prematurity. Most of the infants had been ventilated from birth, but some more mature babies, all with respiratory distress syndrome, had been ventilated because of worsening blood gas tensions. ${ }^{4}$ Ventilator settings before the study were determined by the clinical team caring for the infants. Ventilator rates, except in three infants who were more than 72 hours old, were in excess of 60 breaths/minute. No infant was paralysed or had received sedation.

The 22 infants represented those patients ventilated consecutively with the SLE Newborn 250 ventilator in patient trigger mode. Patient triggered ventilation was started during the acute stage of the infant's illness according to the availability of the ventilator (fig 1).

Infants were entered into the study once they had been stable on conventional ventilation for at least two hours. They were then transferred to continuous positive airways pressure (CPAP) trigger mode. The function of the SLE ventilator in patient triggered mode and the pneumotachograph triggering sensor have previously been described in detail. ${ }^{3}$ Briefly, the pneumotachograph, placed between the endotracheal tube and the ventilator circuit, detects changes in flow and triggering occurs if the inspiratory flow exceeds $0.4 \mathrm{l} /$ minute. If respiratory efforts are insufficient to cause triggering after seven seconds, a manual breath will be automatically delivered.

The inspired oxygen concentration, peak inspiratory pressure and positive end expiratory pressure (PEEP) were not altered on the introduction of the patient trigger mode. A standard flow rate within 


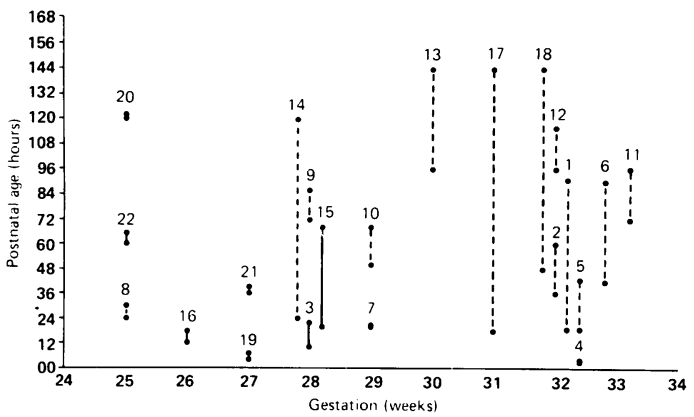

Fig 1 The postnatal and gestational ages of the infants receiving patient triggered ventilation. Each infant is represented by two linked data points, the length of the joining line representing the duration that the ventilation was maintained. Infants in whom it was successfully maintained until extubation are depicted by the interrupted lines, the failures by the solid lines.

the ventilator circuit was used in all infants, $81 /$ minute, and this was kept constant during patient triggered ventilation. The inflation time was altered after observation of the infant's respiratory rate while receiving CPAP for one minute. ${ }^{5}$ From the respiratory rate the inspiratory time was calculated to yield an inspiratory:expiratory (I:E) ratio of $1: 1 \cdot 2$. As a systems delay of up to 40 milliseconds can be experienced, ${ }^{3}$ the inflation time set during patient triggered ventilation was the calculated inspiratory time minus 40 milliseconds.

On starting patient trigger mode the infant's respiratory efforts during ventilation were observed. If the inspiratory efforts were distinct from inflation, evidence of active expiration or asynchrony, ${ }^{6}$ the duration of inflation was reduced in steps to a minimum of 0.1 seconds. Detailed respiratory measurements were then made, simultaneously recording flow, volume, and oesophageal and ventilator pressure changes as previously described. ${ }^{6}$ Infants who, despite reduction in inflation time, remained asynchronous were returned to conventional mode.

Throughout the period of ventilation the infants were continuously monitored using either a Searle intra-arterial electrode or a transcutaneous oxygen monitor (Draeger). Arterial blood gases were sampled from an indwelling umbilical or peripheral arterial catheter. Gases were sampled immediately before and 20 minutes and one hour after, switching to trigger mode. The inspired oxygen concentration was altered to maintain arterial oxygen pressure $\left(\mathrm{PaO}_{2}\right)$ between 6.67-9.33 kPa (50-70 mm $\left.\mathrm{Hg}\right)$. Subsequently arterial blood gases were checked at four hourly intervals, or more frequently if clinically indicated. Either peak inspiratory pressure or inspired oxygen concentration, or both, were reduced in response to improvements.

Patient triggered ventilation was continued until either ventilatory support was no longer required and the infant extubated or patient triggered ventilation 'failed'. It was considered to have failed if: the infant developed a respiratory acidosis $(\mathrm{pH}<7 \cdot 25)$; a metabolic acidosis developed, suggesting fatigue (other causes such as hypothermia, hypovolaemia, and sepsis having been excluded); or the infant became either asynchronous despite reduction in inspiratory time, or developed a pneumothorax.

To determine the cause of the deterioration of blood gases the infant was returned to conventional mode with the pneumotachograph remaining in the airway, and arterial blood gases were estimated 20 minutes later. Improvement indicated that inconsistent triggering had been responsible, no improvement suggested that the original deterioration was a result of the added dead space of the pneumotachograph within the airway. The latter was confirmed if the blood gases improved after a further period on conventional mode with the pneumotachograph removed from the airway.

Inspired oxygen concentrations and rate of delivery of positive pressure inflations during conventional mode were compared with those after one hour on patient triggered ventilation. Differences were assessed for significance by the paired Wilcoxon rank sum test. Comparisons were also made between those infants in whom patient triggered ventilation failed, and those in whom it succeeded. Differences between the groups were statistically assessed by the Wilcoxon rank sum test and Fisher's exact test. This study was approved by the hospital's ethics committee.

\section{Results}

Patient triggered ventilation was maintained for variable times in the 22 infants. The median duration was 19 hours (range 1-240). In all 22 infants the inflation time used was 0.4 seconds or less and in the more immature infants it was as low as 0.2 seconds, median inflation time being 0.32 seconds. Initially all infants were synchronous as determined by clinical observation. ${ }^{6}$ Those in whom long term patient triggered ventilation was successful remained synchronous without further change in the inflation time.

One infant (of 32 weeks' gestation) developed a serious complication during patient triggered ventilation-a pneumothorax-12 hours after starting the trigger mode. The infant became 
Table Inflation time and pressure, gestational age, and changes in inspired oxygen concentrations

\begin{tabular}{|c|c|c|c|c|}
\hline Inflation time (secs) & $\begin{array}{l}\text { Peak inspiratory } \\
\text { pressure }\left(\mathrm{cm} \mathrm{H}_{2} \mathrm{O}\right)\end{array}$ & $\begin{array}{l}\text { Gestational age } \\
\text { (weeks) }\end{array}$ & $\begin{array}{l}\mathrm{FiO}_{2} \text { before } \\
\text { ventilation }(\%)\end{array}$ & $\begin{array}{l}\mathrm{FiO}_{2} \text { one hour } \\
\text { after ventilation (\%) }\end{array}$ \\
\hline \multicolumn{5}{|c|}{ Infants for whom patient triggered ventilation was successful: } \\
\hline 0.4 & 24 & 32 & 60 & 60 \\
\hline $0 \cdot 35$ & 18 & 32 & 45 & 40 \\
\hline $0 \cdot 35$ & 24 & 32 & 70 & 60 \\
\hline $0 \cdot 4$ & 22 & 33 & 50 & 45 \\
\hline 0.4 & 15 & 25 & 30 & 25 \\
\hline $0 \cdot 3$ & 16 & 28 & 30 & 25 \\
\hline $0 \cdot 27$ & 17 & 29 & 40 & 30 \\
\hline $0 \cdot 4$ & 21 & 33 & 40 & 35 \\
\hline $0 \cdot 4$ & 18 & 32 & 40 & 35 \\
\hline $0 \cdot 28$ & 17 & 30 & 65 & 55 \\
\hline $0 \cdot 3$ & 14 & 28 & 55 & 45 \\
\hline $0 \cdot 32$ & 26 & 31 & 50 & 45 \\
\hline $0 \cdot 36$ & 24 & 32 & 50 & 45 \\
\hline \multicolumn{5}{|c|}{ Infants for whom patient triggered ventilation failed: } \\
\hline $0 \cdot 27$ & 16 & 28 & 60 & 40 \\
\hline 0.4 & 24 & 32 & 85 & 95 \\
\hline 0.25 & 24 & 29 & 60 & 60 \\
\hline 0.32 & 32 & 28 & 65 & 75 \\
\hline 0.25 & 15 & 26 & 30 & 21 \\
\hline 0.31 & 18 & 27 & 80 & 80 \\
\hline 0.2 & 24 & 25 & 70 & 75 \\
\hline 0.33 & 19 & 27 & 50 & 40 \\
\hline 0.29 & 18 & 25 & 45 & 35 \\
\hline
\end{tabular}

asynchronous immediately before the development of the airleak. A fault had developed in the expiratory line of the ventilator so that it was impossible to reduce the PEEP below $6 \mathrm{~cm} \mathrm{H}_{2} \mathrm{O}$.

Comparison of arterial blood gases after one hour on patient triggered ventilation with those taken immediately before changing from conventional mode showed an improvement in oxygenation $(p<0.05)$ in most of the infants. Consequently it was possible to reduce the inspired oxygen concentrations from a median of $50 \%$ (range $30-85$ ) to $45 \%$ (range 21-95) $(\mathrm{p}<0.05)$, (table), and maintain arterial oxygen saturation within the desired range. The improvement in oxygenation was also associated with an increase in delivery rate of positive pressure inflations from a median of 60 breaths/ minute (range 20-80) to 72 breaths/minute (48-120), $(\mathrm{p}<0 \cdot 01)$ :

Thirteen infants tolerated patient triggered ventilation until final extubation without complications. The median duration was 24 hours (range 6-240). In the remaining nine babies patient triggered ventilation failed after variable time periods (median duration 3 hours, range 1-48) (fig 1). There was no significant difference between these two groups in the postnatal age at which they started patient triggered ventilation (median 42 hours, range 18-96, and median 20 hours, range 3-120 hours, respectively). The first group, however, were significantly more mature (median 32 weeks, range 28-33) and heavier at birth (median $1600 \mathrm{~g}$, range 650-1910) than the second group (median 27 weeks, range 25-32; median $1080 \mathrm{~g}$, range 560-1910) $(p<0.01)$. The second group also tended to receive shorter inflation times (median 0.29 secs, range $0.2-0.33$ ) than the first (median 0.35 secs, range $0 \cdot 27-0 \cdot 4),(\mathrm{p}<0 \cdot 05)$.

The change to patient triggered ventilation was more often associated with a lack of improvement in oxygenation after the first hour in those infants in whom it finally failed $(p<0.05)$. The inflation rate during patient triggered ventilation tended to be inversely proportional to gestational age in those infants in whom it was successful, and similar to their previously observed spontaneous respiratory rate (fig 2). In six of the infants in whom it failed (all of 28 weeks' gestation or less) the delivery rate of inflations at one hour was at least 20 breaths/minute lower than their spontaneous respiratory rate (fig 2 ).

Two infants whose spontaneous respiratory rates and initial triggering rate were in excess of 100/ minute (fig 2) subsequently failed to trigger the ventilator on about one breath in four. These infants then became asynchronous, and were unresponsive to a reduction in inspiratory time. One infant suddenly failed to trigger the ventilator without 


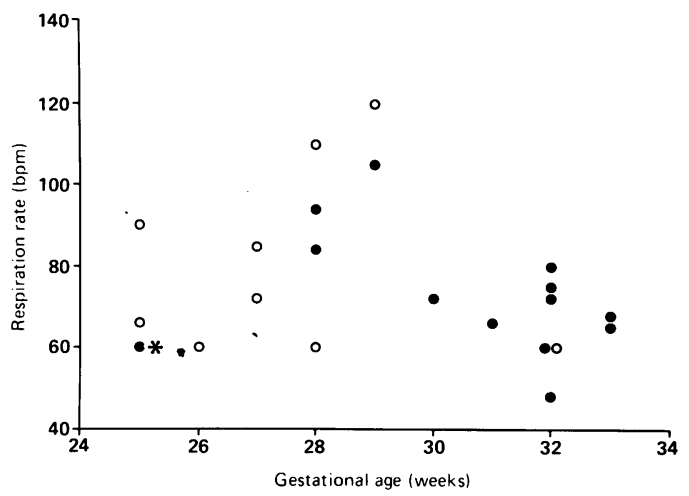

Fig 2 The rate of delivery of positive pressure inflations at one hour related to gestational age during patient triggered ventilation. $=$ those for whom it was successful; $\mathrm{O}=$ those for whom it failed; $*=$ an infant of 25 weeks' gestation in whom patient triggered ventilation was only started during weaning.

obvious cause. The remaining five infants developed a metabolic acidosis after variable periods on patient triggered ventilation. In all cases this reversed after the infant returned to conventional mode, despite the pneumotachograph remaining in the circuit.

\section{Discussion}

This study confirmed that patient triggered ventilation, using airflow changes, was beneficial in most patients. Initially all the infants, on clinical observation, became synchronous ${ }^{6}$ and this explains the improvement in oxygenation. ${ }^{7}$ Those infants in whom long term patient triggered ventilation was successful remained synchronous throughout ventilation and thus did not require sedation or paralysing agents. ${ }^{8}$ Conversion to synchronous respiration and reduction in active expiration is also associated with high frequency positive pressure ventilation (HFPPV). ${ }^{9} \mathrm{~A}$ randomised trial is at present being conducted to assess which of these two modes of ventilation is the more efficacious at preventing this respiratory interaction.

Patient triggered ventilation is successful if the infant is synchronous with the ventilator, that is if the start of positive pressure inflation occurs early in inspiration ${ }^{5}$ and if inflation has ceased before late expiration. ${ }^{10}$ Such timing is dependent on three factors; the duration of the systems delay, the stage during inspiration at which the infant's airflow exceeds the triggering point, and the duration of inflation. The maximum systems delay time of the SLE and pneumotachograph sensor has been shown to be 40 milliseconds. This systems delay time must be subtracted from the calculated inflation time to prevent inflation continuing into expiration. Although in the present study this meant that in some of the more immature infants the inflation time was as short as $\mathbf{0 . 2}$ seconds, oxygenation was maintained. A longer systems delay time, however, would certainly be a disadvantage. ${ }^{11}$ In the present study, as seen previously, ${ }^{3}$ the infants were able to generate the critical change in airflow early in inspiration. This success may be explained as airflow changes tend to be fastest early in inspiration. The infants included in this study all had respiratory distress and were less than one week old, thus airway resistance was likely to be low, which would be expected to facilitate early rapid airflow changes. The duration of inflation time is of crucial importance and must be sufficiently short to prevent inflation persisting into spontaneous expiration. ${ }^{10}$ In this study this was successfully determined from clinical observation of the infants' spontaneous respiratory rates, and as a consequence, in no infant was an inflation time greater than $\mathbf{0 . 4}$ seconds used. This could be predicted from previous studies, which have shown that very preterm infants have a fast spontaneous respiratory rate and hence short inspiration. ${ }^{5}$ Even during weaning, limitation of inspiratory time to 0.5 seconds improves oxygenation and prevents active expiration. ${ }^{12}$

Only one infant developed a pneumothorax during patient triggered ventilation; this low incidence may, however, simply reflect the population studied, and a randomised trial is necessary before it can be claimed that patient triggered ventilation is associated with a reduction in the incidence of air leaks. Although the infant who developed an airleak had become asynchronous immediately before the pneumothorax developed, a simultaneous increase in PEEP was noted, a factor previously incriminated in increasing the incidence of airleaks. ${ }^{13}$ On examination of the ventilator a malfunction of the expiratory valve was noted and this was independent of ventilator mode.

This study has outlined important limitations of patient triggered ventilation among preterm neonates. Disappointingly, in addition to the infant who developed a pneumothorax, patient triggered ventilation was considered to have failed on a further eight occasions. It failed in the more immature infants of less than 28 weeks' gestational age. The only infant of less than 28 weeks' gestation who tolerated patient triggered ventilation till extubation was only started on it during weaning (conventional ventilator rate at 20 breaths/minute), and thus was recovering from respiratory distress.

Failure of patient triggered ventilation was seen in 
two infants whose triggered inflation rates exceeded 100 breaths/minute. Both infants subsequently failed to generate sufficient airflow change to trigger the ventilator consistently and they then became asynchronous. At such high inflation frequencies ventilator function is impaired and, in particular, inadvertent PEEP may occur. ${ }^{14}$ This may have contributed to the change in respiratory effort. A further problem is that until the ventilator responds to the patient's inspiratory efforts-that is, the time taken by the systems delay-the infant's respiration is essentially unsupported and the ventilator is acting not as a ventilator but as a respiratory obstruction, which became more apparent at increased rates. It has been suggested that the response time, or systems delay time, should not exceed $10 \%$ of the total inflation time; a systems delay of 0.036 seconds would permit a maximum ventilator rate of 83 breaths/minute. ${ }^{11}$ As occurred in this study, when triggering was attempted at an appreciably higher rate than that allowed by the response time, incoordination with the ventilator resulted. ${ }^{11}$

Another possible explanation for the failure of patient triggered ventilation in the immature infants was the use of short inflation times in this group. Limitation of inflation time reduces the area under the pressure/time curve for a given breath and decreases the assistance delivered by the ventilator. Ventilator waveform is also affected by a reduction in inflation time ${ }^{15}$ particularly if-as in this studyflow is not increased. This may have compromised the effectiveness of ventilation further. Limitation of inflation time was necessary to prevent asynchrony, and short inflation times were tolerated successfully by some infants to extubation. Increasing peak inspiratory pressure would have increased the mean airway pressure and thus possibly have compensated for the limitation of inflation time. We were reluctant, however, to adopt this approach with its known hazards, ${ }^{16}$ particularly as among nonparalysed infants short inspiratory times, without an increase in peak inspiratory pressure, can be associated with improvements in oxygenation if delivered at fast rates. ${ }^{7}$

Failure of patient triggered ventilation in the remaining infants was associated with the development of a metabolic acidosis. In all cases this disappeared once the infant had been returned to conventional mode, even though the pneumotachograph remained in the circuit. This suggested that the metabolic acidosis was due to fatigue. Although the added deadspace of the pneumotachograph in the airway has previously not been associated with deterioration in blood gas tensions, ${ }^{17}$ among these tiny infants it may have added critically to the work of breathing. This airflow triggering system resulted in synchronous respiration in all infants, thus an advantageous future development would be an alternative method of detection of airflow changes that did not appreciably increase the deadspace of even the smallest infants.

The present results have shown useful early predictors of failure of patient triggered ventilation, that is a failure of improvement in oxygenation and slow triggering rate when related to gestational age. Immature infants with respiratory distress have previously been shown to have a fast spontaneous respiratory rate in the first 48 hours of life. ${ }^{5}$ This was recorded, however, during a very short time period. ${ }^{5}$ It seems likely that very preterm infants, particularly considering the limitations of patient triggered ventilation at fast rates noted above, may be unable to sustain such rapid rates even for one hour during patient triggered mode. The failure of improvement in oxygenation at one hour would then be consequent on the comparatively slow triggering rate. ${ }^{7}$ Our results suggest that any infant who fails to trigger the ventilator at a similar frequency, that is within 20 breaths/minute, to his or her own respiratory rate, or in whom oxygenation fails to improve after one hour on patient triggered ventilation, should be returned to conventional mode. In this group of infants during the acute stage of the illness HFPPV may be more successful, ${ }^{7}$ and patient ${ }^{\circ}$ triggered ventilation may be reserved for weaning. ${ }^{3}$

In summary, patient triggered ventilation using airflow detected at the airway as the triggering mechanism can be an effective form of long term neonatal ventilation, but there are limitations, particularly in the more immature infants. The success or failure of this mode of ventilation is usually apparent within a short time period. Randomised studies are required to determine if patient triggered ventilation, when compared with HFPPV, is advantageous to the preterm neonate in reducing the duration of mechanical ventilation or long term complications.

Dr A Mitchell is supported by the Royal Air Force and Dr M Hird by Children Nationwide Medical Research Fund. The equipment for this study was generously provided by Specialised Laboratory Equipment (SLE) Ltd and Children Nationwide Medical Research Fund. We are grateful to Dr H Gamsu for his continuing support and advice and the enthusiasm of the staff of the neonatal intensive care unit.

\footnotetext{
References

1 Mehta A, Callan K, Wright BM, Stacey TE. Patient triggered ventilation in the newborn. Lancet 1986;ii:706-12.

2 Greenough A, Greenall F. Patient triggered ventilation in premature neonates. Arch Dis Child 1988;63:77-8.

3 Greenough A. Pool J. Neonatal patient triggered ventilation. Arch Dis Child 1988;63:394-7.
} 
${ }^{4}$ Greenough A, Roberton NRC. Morbidity and survival in neonates ventilated for the respiratory distress syndrome. $\mathrm{Br}$ Med J 1985;290:597-600.

5 Greenough A, Greenall F, Gamsu H. Synchronous respiration, which ventilator rate is best? Acta Paediatr Scand 1987;76: 713-6.

${ }^{6}$ Greenough A, Greenall F. Observation of spontaneous respiratory interaction with artificial ventilation. Arch Dis Child 1988;63:168-71.

7 Greenough A, Pool J, Greenall F, Morley C, Gamsu H. Comparison of two different rates of artificial ventilation in premature neonates with RDS. Acta Paediatr Scand 1987;76: 706-12.

${ }^{8}$ Greenough A, Wood S, Morley C, Davis J. Pancuronium prevents pneumothoraces in ventilated premature babies who actively expire against positive pressure inflation. Lancet 1984 ; i:1-4.

9 Greenough A, Morley CJ, Pool J. Fighting the ventilator are fast rates an alternative to paralysis? Early Hum Dev 1986;13: $189-94$.

10 Greenough A. The premature infant's respiratory response to mechanical ventilation. Early Hum Dev 1988;17:1-5.

11 Epstein RA. The sensitivities and response times of ventilatory assists. Anesthesiology 1971;34:321-6.
12 Greenough A, Greenall F, Gamsu H. Inspiratory times when weaning from mechanical ventilation. Arch Dis Child 1987;62: 1269-70.

${ }^{13}$ Berg TJ, Pagtakhan RD, Reed MH, Langston C, Cherniack V. Bronchopulmonary dysplasia and lung rupture in HMD: influence of continuous distending pressure. Pediatrics 1976;55: 51-3.

14 Greenough A, Greenall.F. Performance of respirators at fast rates commonly used in neonatal intensive care units. Pediatr Pulmonol 1987;3:357-361.

15 Boros SJ, Bind DR, Mammel KC, Kegen E, Gardo MJ. Using conventional infant ventilators at unconventional rates. Pediatrics 1984;74:487-92.

16 Taghizadeh A, Reynolds EOR. Pathogenesis of bronchopulmonary dysplasia following hyaline membrane disease. Am J Pathol 1976;82:487-92.

17 Greenough A, Morley CJ, Davis J. Respiratory reflexes in ventilated premature babies. Early Hum Dev 1983;8:65-75.

Correspondence to Dr A Greenough, Department of Child Health, King's College Hospital, Denmark Hill, London SE5 8RX. 